\title{
Stable derived functors, the Steenrod algebra and homological algebra in the category of functors
}

\author{
by \\ Stanisław Betley (Warszawa)
}

\begin{abstract}
We present a very short way of calculating additively the stable (co)homology of Eilenberg-MacLane spaces $K(\mathbb{Z} / p, n)$. Our method depends only on homological algebra in appropriate categories of functors.
\end{abstract}

Introduction. In the present paper we compute the stable homology of the Eilenberg-MacLane space $K(\mathbb{Z} / p, n)$. Of course the answer has been known for years and not the answer but the method is important here. We start our calculation from the point where the problem was approached classically - from the homotopical equality $K(\mathbb{Z}, n)=\mathrm{SP}^{\infty} S^{n}$ and its $\bmod p$ version. This leads to the interpretation of the stable homology of $K(\mathbb{Z} / p, n)$ in terms of stable derived functors in the sense of Dold-Puppe of symmetric power functors (also very classical). But then we use a more modern interpretation of these stable derived functors as Tor groups in the abelian category of functors from sets to $\mathbb{Z} / p$-vector spaces. Then surprisingly the main result is derived from the properties of Koszul and de Rham sequences relating symmetric and exterior power functors.

There are several reasons for writing this note. First of all we present a very short "stable" calculation of the stable homology of $K(\mathbb{Z} / p, n)$. Classically calculations followed the following route: first one had to do them unstably for all $n$ and then to analyse the stabilization process to check which classes survive. It was always lengthy and complicated. Here we skip the unstable part. It seems to us that this point of view on the Steenrod algebra can be fruitful and we are going to explore it in further papers. Secondly we show that the "shape" of the Steenrod algebra (dimensions of

2000 Mathematics Subject Classification: Primary 55P20; Secondary 55S10, 55U99, $18 \mathrm{G} 15$.

The author was partially supported by the Polish Scientific Grant (KBN) 2 P03A 00218. 
nontrivial groups and their gradation) is determined directly by the existence of the Frobenius morphism, that is, by the fact that raising to the $p$ th power is additive. This has an interesting philosophical meaning that "all" special properties of theories over fields of finite characteristic are encoded in the existence of the Frobenius morphism. The same point of view was promoted in $[\mathrm{BS}]$ where we studied the behaviour of the Goodwillie tower of the identity functor at the prime $p$.

In [B1] and [B2] Bousfield studied the stable derived functors of symmetric powers and the algebra structure induced on them by the composition multiplication of the functor $\sum_{i=1}^{\infty} \mathrm{SP}^{i}$ (here $\mathrm{SP}^{i}$ is the $i$ th symmetric power functor). The main motivation for our work was to understand these two preprints and their relations to the Steenrod algebra. Our paper gives only an additive calculation of $H_{*}^{\text {st }}(K(\mathbb{Z} / p, n), \mathbb{Z} / p)$ but in the last section we show how to approach the multiplicative structure of the Steenrod algebra using our methods. We hope to get Adem relations using similar arguments. Then we will be in a position to study the Steenrod algebra by a new method.

The paper is written in the homological setting mostly because it grew out of an attempt to understand [B1] and [B2] which were written "homologically". On the other hand switching to cohomology is not complicated: see 4.13 .

1. Stable homology of $K(\mathbb{Z} / p, n)$ and stable derived functors. In this section we are perhaps a little sketchy but most of the material here is "classical" and can be found in [DP], [DT], [B1], [B2] or related papers. Let $T: R$-modules $\rightarrow R$-modules be a functor; we shall use the same letter for the degreewise extension of $T$ to the simplicial $R$-modules. Dold and Puppe [DP] defined the $i$ th stable derived functor $L_{i}^{\text {st }} T(\cdot)$ of $T$ by the formula

$$
L_{i}^{\mathrm{st}} T(A)=\lim _{n} \pi_{i+n} T\left(\widetilde{R}\left[S^{n}\right] \otimes P_{*}\right)
$$

where $S^{n}$ denotes any simplicial model for the $n$-dimensional sphere, $P_{*}$ is a projective resolution of $A$ in $R$-modules, the limit is taken with respect to the suspension of spheres, and $\widetilde{R}\left[S^{n}\right]=R\left[S^{n}\right] / R[*]$.

Let $\mathcal{V}_{p}$ denote the category of vector spaces over $\mathbb{Z} / p$. For a functor $T: \mathcal{V}_{p} \rightarrow \mathcal{V}_{p}$ we have the formula

$$
L_{i}^{\mathrm{st}} T(\mathbb{Z} / p)=\lim _{n} \pi_{i+n} T\left(\widetilde{\mathbb{Z}} / p\left[S^{n}\right]\right)
$$

and these groups are often called (see $[\mathrm{P}]$ ) the stable homotopy of the functor $T \circ \widetilde{\mathbb{Z}} / p[\cdot]$ which is defined on the category of simplicial sets and takes values in simplicial objects over $\mathcal{V}_{p}$.

Let $\mathrm{SP}^{i}: \mathcal{V}_{p} \rightarrow \mathcal{V}_{p}$ be the $i$ th symmetric power functor and write $\mathrm{SP}^{*}=$ $\sum_{i=1}^{\infty} \mathrm{SP}^{i}$. Then $\mathrm{SP}^{*}$ is the ordinary polynomial algebra functor. If we divide it by the ideal generated by the image of the Frobenius morphism $\mathrm{SP}^{1} \rightarrow$ 
$\mathrm{SP}^{p}$ given by raising to the $p$ th power then we obtain a graded functor $\mathrm{SP}_{p}^{*}=\sum_{i=1}^{\infty} \mathrm{SP}_{p}^{i}$ which we call reduced symmetric powers.

We need one more piece of notation. Let $H_{i}^{\text {st }}(K(\mathbb{Z}), \mathbb{Z} / p)$ denote the group $\lim _{n} H_{i+n}(K(\mathbb{Z}, n), \mathbb{Z} / p)$ where the limit is taken in the ordinary way which gives the stable homology of an Eilenberg-MacLane space. Similarly

$$
H_{i}^{\mathrm{st}}(K(\mathbb{Z} / p), \mathbb{Z} / p)=\lim _{n} H_{i+n}(K(\mathbb{Z} / p, n), \mathbb{Z} / p) .
$$

1.1. Lemma. We have the following formulas:

$$
\begin{aligned}
& L_{*}^{\text {st }} \operatorname{SP}^{*}(\mathbb{Z} / p)=H_{*}^{\text {st }}(K(Z), \mathbb{Z} / p), \\
& L_{*}^{\text {st }} \operatorname{SP}_{p}^{*}(\mathbb{Z} / p)=H_{*}^{\text {st }}(K(\mathbb{Z} / p), \mathbb{Z} / p) .
\end{aligned}
$$

Proof. By the famous Dold-Thom theorem from [DT] we can use $\mathrm{SP}^{\infty} S^{n}$ as a model for the space $K(\mathbb{Z}, n)$. Let us remind the reader that we work in the category of simplicial sets and $\mathrm{SP}^{\infty} S^{n}$ denotes the infinite symmetric product of the simplicial model of the $n$-dimensional sphere. Then we have an isomorphism of simplicial abelian groups

$$
\widetilde{\mathbb{Z}} / p\left[\mathrm{SP}^{\infty} S^{n}\right] \simeq \sum_{i=1}^{\infty} \mathrm{SP}^{i}\left(\widetilde{\mathbb{Z}} / p\left[S^{n}\right]\right)
$$

which we obtain by working directly with bases of both groups (both groups are free over $\mathbb{Z} / p$ ). The homotopy groups of the left hand side give us the homology of $K(\mathbb{Z}, n)$ with $\mathbb{Z} / p$ coefficients. It is easy to see that the isomorphism 1.2 commutes with the suspension map. This follows from the fact that 1.2 can be stated in the form $\widetilde{\mathbb{Z}} / p\left[\mathrm{SP}^{\infty} X\right] \simeq \sum_{i=1}^{\infty} \mathrm{SP}^{i}(\widetilde{\mathbb{Z}} / p[X])$ for any simplicial set $X$ and this isomorphism is natural in $X$. Hence after stabilization we get the first formula of our lemma.

The proof for reduced symmetric powers $\mathrm{SP}_{p}^{i}$ goes similarly. Let $X$ be a pointed set with base point $e$. For $x \in \mathrm{SP}^{\infty} X$ define $x_{i_{1}, \ldots, i_{k}}$ as the point in $\mathrm{SP}^{\infty} X$ obtained from $x$ by putting $e$ on coordinates $i_{1}, \ldots, i_{k}$.

Define the reduced infinite symmetric product of $X$ as

$$
\mathrm{SP}_{p}^{\infty} X=\mathrm{SP}^{\infty} X / \sim
$$

where $x \sim y$ if for $x=\left(x_{1}, x_{2}, \ldots\right)$ and $y=\left(y_{1}, y_{2}, \ldots\right)$ we have $i_{1}, \ldots, i_{p}$ and $j_{1}, \ldots, j_{p}$ such that $x_{i_{a}}=x_{i_{b}}$ for $a, b=1, \ldots, p$, the same is true for $y$ on coordinates $j_{1}, \ldots, j_{p}$, and $x_{i_{1}, \ldots, i_{p}}=y_{j_{1}, \ldots, j_{p}}$. In other words we identify $x$ as above with the point which has $e$ on coordinates $i_{1}, \ldots, i_{p}$. Observe that $\mathrm{SP}_{p}^{\infty} X$ is an abelian $p$ torsion group for any set $X$. This observation extends to simplicial sets and one gets a simplicial abelian $p$-torsion group associated with any simplicial set.

Claim. $\operatorname{SP}_{p}^{\infty} X \simeq \widetilde{\mathbb{Z}} / p[X]$. 
Proof. If we write an element of $\mathrm{SP}_{p}^{\infty} X$ as a tuple $\left(x_{1}^{i_{1}}, \ldots, x_{n}^{i_{n}}\right)$ by writing $\left(x, \ldots, x, y_{1}, \ldots\right)$ as $\left(x^{i}, y_{1}, \ldots\right)$ and skipping $e$ from the notation then we see that the map

$$
\left(x_{1}^{i_{1}}, \ldots, x_{n}^{i_{n}}\right) \mapsto i_{1} \cdot x_{1}+\ldots+i_{n} \cdot x_{n}
$$

is a group isomorphism. The proof of 1.1 is finished by the observation that $\widetilde{\mathbb{Z}} / p\left[S^{n}\right]=K(\mathbb{Z} / p, n)$, the isomorphism from the claim commutes with suspension and we can proceed as in 1.2 with reduced powers and reduced symmetric products.

1.3. Remark. From the definition of stable derived functors we also get

$$
H_{*}^{\mathrm{st}}(K(\mathbb{Z}), \mathbb{Z} / p)=L_{*}^{\mathrm{st}}(\widetilde{\mathbb{Z}} / p[\cdot])(\mathbb{Z})
$$

and

$$
H_{*}^{\mathrm{st}}(K(\mathbb{Z} / p), \mathbb{Z} / p)=L_{*}^{\mathrm{st}}(\widetilde{\mathbb{Z}} / p[\cdot])(\mathbb{Z} / p)
$$

where we treat $\widetilde{\mathbb{Z}} / p[\cdot]$ as a functor $\mathrm{Ab} \rightarrow \mathrm{Ab}$. In the theory of stable derived functors we have the universal coefficients theorem which gives the exact sequence for any abelian group $A$ :

$$
0 \rightarrow L_{i}^{\text {st }} T(\mathbb{Z}) \otimes A \rightarrow L_{i}^{\text {st }} T(A) \rightarrow \operatorname{Tor}\left(L_{i-1}^{\text {st }} T(\mathbb{Z}), A\right) \rightarrow 0 .
$$

So we know that $L_{*}^{\text {st }}(\widetilde{\mathbb{Z}} / p[\cdot])(\mathbb{Z})=L_{*}^{\text {st }}(\widetilde{\mathbb{Z}} / p[\cdot])(\mathbb{Z}) \otimes \mathbb{Z} / p$ embeds into $L_{*}^{\text {st }}(\widetilde{\mathbb{Z}} / p[\cdot])(\mathbb{Z} / p)=H_{*}^{\text {st }}(K(\mathbb{Z} / p), \mathbb{Z} / p)$. It is easy to see that this embedding is induced by the quotient map $\mathrm{SP}^{*} \rightarrow \mathrm{SP}_{p}^{*}$. We will return to this point later.

\section{Stable derived functors and homological algebra in the cate-} gory of functors. Let $\Gamma$ denote the category of functors from finite pointed sets to $\mathcal{V}_{p}$. Following Pirashvili $[\mathrm{P}]$ we denote by $t^{*}$ the functor $X \mapsto \widetilde{\mathbb{Z}} / p[X]$ and by $t$ its dual. As previously we use the same letter for the functor and its standard degreewise extension to simplicial sets. Moreover if $T: \mathcal{V}_{p} \rightarrow \mathcal{V}_{p}$ is a functor then we use the same letter for $T \circ t^{*}$ as an element of $\Gamma$. We have:

2.1. Lemma ([P, Proposition 2.2]). If $T: \mathcal{V}_{p} \rightarrow \mathcal{V}_{p}$ then

$$
L_{i}^{\mathrm{st}} T(\mathbb{Z} / p)=\operatorname{Tor}_{i}^{\Gamma}(t, T) .
$$

This means that trying to calculate $L_{i}^{\text {st }} \mathrm{SP}^{*}$ we are forced to calculate Tor groups in the category of functors. Before turning to calculations we give some simple lemmas which make such calculations easier.

2.2. Lemma ([P, Lemma 4.2]). Let $S \in \Gamma$ and $T \in \Gamma$ satisfy $S(*)=$ $T(*)=0$, where $*$ denotes the one-point set. Then $\operatorname{Tor}_{*}^{\Gamma}(t, S \otimes T)=0$.

Proof. This follows immediately from the Eilenberg-Zilber and Künneth formulas. 


\subsection{LEMMA.}

$$
\operatorname{Tor}_{0}^{\Gamma}\left(t, t^{*}\right)=\mathbb{Z} / p, \quad \operatorname{Tor}_{i}^{\Gamma}\left(t, t^{*}\right)=0 \quad \text { for } i>0 .
$$

Proof. By 2.1 we know that $\operatorname{Tor}_{i}^{\Gamma}\left(t, t^{*}\right)=L_{i}^{\text {st }} \operatorname{Id}(\mathbb{Z} / p)$. By the definition of stable derived functors it follows that the groups $\operatorname{Tor}_{*}^{\Gamma}\left(t, t^{*}\right)$ are the same as the stable homotopy groups of the Eilenberg-MacLane space $K(\mathbb{Z} / p, n)$.

2.4. LEMmA. The Frobenius $F: \mathrm{SP}^{p^{i}} \rightarrow \mathrm{SP}^{p^{i+1}}$ induces a trivial map $\operatorname{Tor}_{i}^{\Gamma}\left(t, \mathrm{SP}^{p^{i}}\right) \rightarrow \operatorname{Tor}_{i}^{\Gamma}\left(t, \mathrm{SP}^{p^{i+1}}\right)$.

Proof. The lemma follows immediately from 2.2 and the fact that the natural transformation of functors $F: \mathrm{SP}^{p^{i}} \rightarrow \mathrm{SP}^{p^{i+1}}$ factors through $\left(\mathrm{SP}^{p^{i}}\right)^{\otimes p}$. This we achieve by the following simple observation. If Id : $\mathcal{V}_{p} \rightarrow \mathcal{V}_{p}$ denotes the identity functor then we have a natural transformation (embedding) $\psi: \mathrm{Id} \rightarrow \mathrm{Id}^{\otimes k}$ for any natural $k$. Here we treat $\mathrm{Id}$ and $\mathrm{Id}^{\otimes k}$ as objects of $\Gamma$. We define this natural transformation on basis elements by the formula $x \mapsto x^{\otimes k}$ and then extend by linearity. Warning: $\psi$ is not a natural transformation of functors $\mathcal{V}_{p} \rightarrow \mathcal{V}_{p}$.

2.5. Lemma. Let $K$ denote the kernel of the quotient morphism $\mathrm{SP}^{p^{i}} \rightarrow$ $\mathrm{SP}_{p}^{p^{i}}$. Then the Frobenius $\mathrm{SP}^{p^{i-1}} \rightarrow \mathrm{SP}^{p^{i}}$ factors uniquely through $K$ and the resulting map $g: \mathrm{SP}^{p^{i-1}} \rightarrow K$ induces an isomorphism $\operatorname{Tor}_{*}^{\Gamma}\left(t, \mathrm{SP}^{p^{i-1}}\right) \rightarrow$ $\operatorname{Tor}_{i}^{\Gamma}(t, K)$.

Proof. The first part is obvious. The image of the Frobenius certainly goes to zero in $\mathrm{SP}_{p}^{p^{i}}$ so the map $g$ exists and is unique from the definition of $K$. But we have more functors with natural maps to $K$. For any $j=$ $0, \ldots, p^{i-1}-1$ we have a map $L_{j}:=\mathrm{SP}^{p^{i-1}-j} \otimes \mathrm{SP}^{j p} \rightarrow \mathrm{SP}^{p^{i}}$ given by multiplication of the Frobenius on $\mathrm{SP}^{p^{i-1}-j}$ and the identity on $\mathrm{SP}^{j p}$. The image of $L_{j}$ is obviously contained in $K$. Let $K_{j}$ be the image of $L_{0} \oplus$ $L_{1} \oplus \ldots \oplus L_{j}$ in $K$. Then the $K_{j}$ 's form a filtration in $K$ with the property that $K_{s} / K_{s-1} \simeq \mathrm{SP}^{p^{i-1}-s} \otimes \mathrm{SP}_{p}^{s p}, K_{0}=\mathrm{SP}^{p^{i-1}}$ and $K_{p^{i-1}-1}=K$. The embedding $K_{s} \rightarrow K_{s+1}$ induces an isomorphism on the corresponding Tor groups by 2.2 so the proof is finished.

3. Preliminary calculations. As the main tool for our calculations we shall use Koszul and de Rham sequences and their properties ([FLS, Section 3]). Let $\mathcal{F}$ denote the category of functors $\mathcal{V}_{p} \rightarrow \mathcal{V}_{p}$. We have the Koszul exact sequence in $\mathcal{F}$ for any $n$ :

$$
0 \rightarrow \Lambda^{n} \rightarrow \Lambda^{n-1} \otimes \mathrm{SP}^{1} \rightarrow \ldots \rightarrow \mathrm{SP}^{n} \rightarrow 0
$$

and the de Rham sequence

$$
0 \leftarrow \Lambda^{n} \leftarrow \Lambda^{n-1} \otimes \mathrm{SP}^{1} \leftarrow \ldots \leftarrow \mathrm{SP}^{n} \leftarrow 0
$$


which in case $n=p k$ has as homology the functors appearing in the sequence

$$
0 \leftarrow \Lambda^{k} \leftarrow \Lambda^{k-1} \otimes \mathrm{SP}^{1} \leftarrow \ldots \leftarrow \mathrm{SP}^{k} \leftarrow 0
$$

with $\mathrm{SP}^{k}$ giving homology at the place corresponding to $\mathrm{SP}^{n}$.

3.1. LeMmA. If $n$ is not a power of $p$ then $\operatorname{Tor}_{*}^{\Gamma}\left(t, \mathrm{SP}^{n}\right)=0$.

Proof (see [FLS, Proposition 6.1]). Observe that if $n$ is not a power of $p$ then there exist two natural numbers $a$ and $b$ such that $a+b=n$ and the index of $\Sigma_{a} \times \Sigma_{b}$ in $\Sigma_{n}$ is not divisible by $p$. But then $\mathrm{SP}^{n}$ is contained as a direct summand in $\mathrm{SP}^{a} \otimes \mathrm{SP}^{b}$ and we can use 2.2 .

Hence we are really interested only in the functors $\mathrm{SP}^{n}$ and $\mathrm{SP}_{p}^{n}$ for $n=p^{k}$. In this section we only deal with the case $k=1$.

3.2. Proposition. The groups $\operatorname{Tor}_{*}^{\Gamma}\left(t, \mathrm{SP}^{p}\right)$ are $\mathbb{Z} / p$ in dimensions $2 i(p-1)$ and $2 i(p-1)+1$ for $i=1,2, \ldots$ and are trivial in other dimensions. The groups $\operatorname{Tor}_{*}^{\Gamma}\left(t, \mathrm{SP}_{p}^{p}\right)$ are $\mathbb{Z} / p$ in dimensions $1,2 i(p-1)$ and $2 i(p-1)+1$ and are trivial in other dimensions.

Proof. We first deal with the functor $\mathrm{SP}^{p}$. Let $K$ denote the Koszul sequence

$$
0 \rightarrow \Lambda^{p} \rightarrow \Lambda^{p-1} \otimes \mathrm{SP}^{1} \rightarrow \ldots \rightarrow \mathrm{SP}^{p} \rightarrow 0
$$

and $R$ the de Rham sequence

$$
0 \leftarrow \Lambda^{p} \leftarrow \Lambda^{p-1} \otimes \mathrm{SP}^{1} \leftarrow \ldots \leftarrow \mathrm{SP}^{p} \leftarrow 0 .
$$

We calculate the hyperhomology spectral sequences ${ }^{j} E_{r, s}(K)$ and ${ }^{j} E_{r, s}(R)$ for the functor $t \otimes_{\Gamma}(\cdot)$ with coefficients in the Koszul and de Rham complexes respectively. The numbers $j=1,2$ denote here which filtration is taken into account: 1 says that we first take homology in the direction of the coefficients and then vertically, while 2 stands for the opposite.

The sequences ${ }^{j} E_{r, s}(K)$ converge to 0 because the Koszul sequence is exact. Then ${ }^{2} E_{r, s}(K)$ and 2.2 give us the formula

$$
\operatorname{Tor}_{i}^{\Gamma}\left(t, \Lambda^{p}\right)=\operatorname{Tor}_{i+p-1}^{\Gamma}\left(t, \mathrm{SP}^{p}\right) .
$$

Observe that the homology of $R$ is nontrivial only in dimensions $p-1$ and $p$ and nontrivial functors are equal to $\mathrm{Id}=\mathrm{SP}^{1}$ by the general properties of de Rham complexes. Hence by 2.3 the spectral sequence ${ }^{1} E_{r, s}^{2}(R)$ has only two nonzero groups at $(p-1,0)$ and $(p, 0)$ and all differentials are trivial for dimensional reasons. So ${ }^{2} E_{r, s}(R)$ converges to two groups isomorphic to $\operatorname{Tor}_{0}^{\Gamma}\left(t, t^{*}\right)=\mathbb{Z} / p$ which appear in dimensions $p-1$ and $p$. Observe that by $2.2,{ }^{2} E_{r, s}^{1}(R)$ has only two possibly nontrivial columns and we have

$$
{ }^{2} E_{0, s}^{1}(R)=\operatorname{Tor}_{s}^{\Gamma}\left(t, \Lambda^{p}\right), \quad{ }^{2} E_{p, s}^{1}(R)=\operatorname{Tor}_{s}^{\Gamma}\left(t, \mathrm{SP}^{p}\right) .
$$


Then by 3.3 and ${ }^{1} E_{r, s}(R)$-calculations we get

$$
\operatorname{Tor}_{p-1}^{\Gamma}\left(t, \Lambda^{p}\right)=\operatorname{Tor}_{p}^{\Gamma}\left(t, \Lambda^{p}\right)=\mathbb{Z} / p,
$$

which implies (again by 3.3) that

$$
\operatorname{Tor}_{2(p-1)}^{\Gamma}\left(t, \mathrm{SP}^{p}\right)=\operatorname{Tor}_{2 p-1}^{\Gamma}\left(t, \mathrm{SP}^{p}\right)=\mathbb{Z} / p .
$$

Then we easily get our conclusion from the fact that we know to which groups ${ }^{2} E_{r, s}(R)$ converges.

Now observe that we have the following exact sequence of functors:

$$
0 \rightarrow \mathrm{Id} \rightarrow \mathrm{SP}^{p} \rightarrow \mathrm{SP}_{p}^{p} \rightarrow 0 .
$$

Taking Tor groups converts a short exact sequence of functors into a long exact sequence of Tor groups. Hence we get our result on $\mathrm{SP}_{p}^{p}$ from the $\mathrm{SP}^{p}$-case using 2.4.

3.5. REMARK. It is not difficult to show using [B2] that the elements obtained from $\operatorname{Tor}_{i}^{\Gamma}\left(t, \mathrm{SP}_{p}^{p}\right)$ correspond to the ordinary generators of the Steenrod algebra (or rather their duals).

4. General calculations. Actually we have to confess that the spectral sequence machinery in Section 3 was not necessary and was used for two secondary reasons. First of all we were very much stimulated by [FLS] and this machinery was the main tool there. Secondly, spectral sequences used in the proof of 3.2 are very simple, contain a lot of zeros and it is easy to see their limits. On the other hand for higher powers of $p$ it is easier to proceed through a more careful analysis of de Rham complexes. But first recall that we want to calculate all groups $\operatorname{Tor}_{i}^{\Gamma}\left(t, \mathrm{SP}_{p}^{p^{n}}\right)$ for any $n$. We get an inductive formula for them below. Until 4.12 we assume that $p \neq 2$.

4.1. LEMMA.

$$
\operatorname{Tor}_{i}^{\Gamma}\left(t, \mathrm{SP}_{p}^{p^{n}}\right)=\operatorname{Tor}_{i}^{\Gamma}\left(t, \mathrm{SP}^{p^{n}}\right) \oplus \operatorname{Tor}_{i-1}^{\Gamma}\left(t, \mathrm{SP}^{p^{n-1}}\right) .
$$

Proof. This follows immediately from 2.5 and the exact sequence

$$
0 \rightarrow K \rightarrow \mathrm{SP}^{p^{n}} \rightarrow \mathrm{SP}_{p}^{p^{n}} \rightarrow 0
$$

Hence it is enough to get our formula for $\operatorname{Tor}_{i}^{\Gamma}\left(t, \mathrm{SP}^{p^{n}}\right)$.

4.2. Lemma.

$$
\operatorname{Tor}_{i}^{\Gamma}\left(t, \Lambda^{p^{n}}\right)=\operatorname{Tor}_{i+p^{n}-1}^{\Gamma}\left(t, \mathrm{SP}^{p^{n}}\right) .
$$

Proof. This follows immediately from the exactness of the Koszul sequence relating $\Lambda^{p^{n}}$ and $\mathrm{SP}^{p^{n}}$ (compare 3.3).

4.3. LEMMA.

$$
\begin{aligned}
& \operatorname{Tor}_{i}^{\Gamma}\left(t, \Lambda^{p^{n}}\right) \\
& \quad=\operatorname{Tor}_{i-p^{n}+1}^{\Gamma}\left(t, \mathrm{SP}^{p^{n}}\right) \oplus \operatorname{Tor}_{i-p^{n}}^{\Gamma}\left(t, \mathrm{SP}^{p^{n-1}}\right) \oplus \operatorname{Tor}_{i-p^{n}+p^{n-1}}^{\Gamma}\left(t, \Lambda^{p^{n-1}}\right) .
\end{aligned}
$$


Before proving 4.3 we give some consequences of this crucial lemma. It allows us to calculate the groups $\operatorname{Tor}_{i}^{\Gamma}\left(t, \mathrm{SP}^{p^{n}}\right)$ inductively.

\subsection{Proposition.}

$$
\operatorname{Tor}_{i}^{\Gamma}\left(t, \mathrm{SP}^{p^{n}}\right)=\bigoplus_{k=0}^{n} \operatorname{Tor}_{i-2 p^{n}+2}^{\Gamma}\left(t, \mathrm{SP}^{p^{k}}\right) \oplus \bigoplus_{k=0}^{n} \operatorname{Tor}_{i-2 p^{n}+1}^{\Gamma}\left(t, \mathrm{SP}^{p^{k-1}}\right) .
$$

Proof. From the Koszul exact sequence relating $\Lambda^{p^{n}}$ and $\mathrm{SP}^{p^{n}}$ we get $\operatorname{Tor}_{i}^{\Gamma}\left(t, \Lambda^{p^{n}}\right)=\operatorname{Tor}_{i+p^{n}-1}^{\Gamma}\left(t, \mathrm{SP}^{p^{n}}\right)$. From this we observe that to make our calculation we have to subtract $p^{n}-1$ from the indices on the right hand side of 4.3. Then we apply 4.3 again and again for the last summand (the group $\left.\operatorname{Tor}_{*}^{\Gamma}\left(t, \Lambda^{k}\right)\right)$. On every step we lower the exterior power by 1 and we produce two new Tor groups with symmetric powers inside. When we achieve $k=0$ we get precisely the desired formula.

4.5. Remark. Observe that formula 4.4 gives us the expected result. By 1.1, 1.2 and 2.1 we know that $H_{*}^{\text {st }}(K(\mathbb{Z}), \mathbb{Z} / p)=\bigoplus_{i=0}^{\infty} \operatorname{Tor}_{*}^{\Gamma}\left(t, \mathrm{SP}^{p^{i}}\right)$. On the other hand by Milnor's calculations we know that additively

$$
\bigoplus_{i=0}^{\infty} \operatorname{Tor}_{*}^{\Gamma}\left(t, \mathrm{SP}^{p^{i}}\right)=\mathbb{Z} / p\left[\xi_{1}, \xi_{2}, \ldots\right] \otimes \Lambda\left(\tau_{1}, \tau_{2}, \ldots\right)
$$

where $\xi_{i}$ and $\tau_{i}$ have degrees $2 p^{i}-2$ and $2 p^{i}-1$ respectively for $i>0$. Let $B_{n}=\mathbb{Z} / p\left[\xi_{1}, \ldots, \xi_{n}\right] \otimes \Lambda\left(\tau_{1}, \ldots, \tau_{n}\right)$ and $C_{n}=\bigoplus_{i=0}^{n} \operatorname{Tor}_{*}^{\Gamma}\left(t, \mathrm{SP}^{p^{i}}\right)$. Then the $B_{n}$ (resp. $C_{n}$ ) form filtrations of the right (resp. left) hand side of 4.6. We show that $C_{n} \simeq B_{n}$ as graded vector spaces over $\mathbb{Z} / p$. To see this observe that for the $i$ th gradation $B_{n}^{i}$ of $B_{n}$ we have the following formula:

$$
B_{n}^{i}=B_{n-1}^{i} \oplus B_{n}^{i-2 p^{n}+2} \oplus B_{n-1}^{i-2 p^{n}+1}
$$

where the second term on the right stands for the elements which are multiplied by $\xi_{n}$ and the third is formed by the elements which are contained in $\mathbb{Z} / p\left[\xi_{1}, \ldots, \xi_{n-1}\right] \otimes \Lambda\left(\tau_{1}, \ldots, \tau_{n}\right)$. But from 4.4 we see that the same formula holds for $C_{n}$. Moreover 3.2 tells us that $B_{1}=\operatorname{Tor}_{*}^{\Gamma}\left(t, \mathrm{SP}^{p}\right)=C_{1}$.

Proof of 4.3. We now have to look a little more carefully on the de Rham complex

$$
0 \rightarrow \mathrm{SP}^{p^{n}} \rightarrow \mathrm{SP}^{p^{n}-1} \otimes \Lambda^{1} \rightarrow \ldots \rightarrow \Lambda^{p^{n}} .
$$

Let $d_{i}$ denote the differential issuing from $\mathrm{SP}^{p^{n}-i} \otimes \Lambda^{i}$. Let $K=$ $\operatorname{ker} d_{p^{n-1}}, I=\operatorname{im} d_{0}$ and $L=\operatorname{im} d_{p^{n-1}-1}$.

4.7. Claim.

$$
\operatorname{Tor}_{i}\left(t, \Lambda^{p^{n}}\right)=\operatorname{Tor}_{i-p^{n}+p^{n-1}}(t, K)
$$


Indeed, by a general formula for the homology of the de Rham complex we know that the sequence

$$
0 \rightarrow K \rightarrow \mathrm{SP}^{p^{n}-p^{n-1}} \otimes \Lambda^{p^{n-1}} \rightarrow \ldots \rightarrow \Lambda^{p^{n}} \rightarrow 0
$$

is exact and we can use 2.2 again to obtain 4.7 .

4.8. Claim.

$$
\operatorname{Tor}_{i}(t, L)=\operatorname{Tor}_{i-p^{n-1}+1}(t, I) .
$$

Let us look at the sequence

$$
0 \rightarrow I \rightarrow \mathrm{SP}^{p^{n}-1} \otimes \Lambda^{1} \rightarrow \ldots \rightarrow \mathrm{SP}^{p^{n}-p^{n-1}+1} \otimes \Lambda^{p^{n-1}-1} \rightarrow L \rightarrow 0 .
$$

The argument here is similar to the proof of 4.7. Of course now the sequence under consideration is not exact. On the other hand all functors $F$ appearing as its homology give trivial $\operatorname{Tor}_{*}^{\Gamma}(t, F)$ groups by 2.2 .

4.9. Claim.

$$
\operatorname{Tor}_{i}(t, K)=\operatorname{Tor}_{i}(t, L) \oplus \operatorname{Tor}_{i}\left(t, \Lambda^{p^{n-1}}\right) .
$$

We have an exact sequence

$$
0 \rightarrow L \rightarrow K \rightarrow \Lambda^{p^{n-1}} \rightarrow 0
$$

by the general description of the homology of the de Rham complex. So it is enough to show (for example) that the epimorphism $K \rightarrow \Lambda^{p^{n-1}}$ splits. We have a natural morphism of functors (defined on finite pointed sets)

$$
f: \Lambda^{p^{n-1}} \rightarrow \mathrm{SP}^{p^{n}-p^{n-1}} \otimes \Lambda^{p^{n-1}}
$$

given on basis elements by

$$
x_{1} \wedge x_{2} \wedge \ldots \wedge x_{p^{n-1}} \mapsto x_{1}^{p^{n-1}-1} x_{2}^{p^{n-1}-1} \ldots x_{p^{n-1}}^{p^{n-1}-1} \otimes x_{1} \wedge x_{2} \wedge \ldots \wedge x_{p^{n-1}}
$$

The differential $d$ in the de Rham complex is given by comultiplication on the symmetric part followed by multiplication on the exterior part. Hence we have the following formula for $d: \mathrm{SP}^{k} \otimes \Lambda^{j} \rightarrow \mathrm{SP}^{k-1} \otimes \Lambda^{j+1}$ in terms of bases:

$$
d\left(x_{1} \cdot \ldots \cdot x_{k} \otimes w\right)=\sum_{i=1}^{k}\left(\left(\prod_{s \in I_{i}} x_{s}\right) \otimes x_{i} \wedge w\right)
$$

where $w$ is an arbitrary exterior element and $I_{i}$ denotes the set $\{1, \ldots, k\}$ with $i$ removed. From this formula one sees immediately that the image of $f$ is contained in $K$ because the exterior product of an element by itself vanishes. So it is enough to show that the intersection of the image of $f$ and $L$ is 0 . We show that none of the basis vectors

$$
a=x_{1}^{p^{n-1}-1} x_{2}^{p^{n-1}-1} \ldots x_{p^{n-1}}^{p^{n-1}-1} \otimes x_{1} \wedge x_{2} \wedge \ldots \wedge x_{p^{n-1}}
$$


is contained in the image of $d$. The simple generalization of the argument to linear combinations is left to the reader. Observe that if $b$ is a basis vector then $d(b)$ is a linear combination of basis vectors which are built up from the same variables as $b$ was. Hence if $a$ is in the image of $d$ then there exists a basis vector $b$ such that one of the pieces of the sum expansion of $d(b)$ is a scalar multiple of $a$. So $a$ and $b$ are written in terms of the same variables. From the formula $(*)$ we find that up to multiplication by a scalar and reordering the variables, $b$ must be of the form

$$
b=x_{1}^{p^{n-1}} x_{2}^{p^{n-1}-1} \ldots x_{p^{n-1}}^{p^{n-1}-1} \otimes x_{2} \wedge \ldots \wedge x_{p^{n-1}} .
$$

But this is impossible because by $(*)$ we see that for such a $b, d(b)=0$.

4.10. Claim.

$$
\operatorname{Tor}_{i}(t, I)=\operatorname{Tor}_{i}\left(t, \mathrm{SP}^{p^{n}}\right) \oplus \operatorname{Tor}_{i-1}\left(t, \mathrm{SP}^{p^{n-1}}\right) .
$$

Again by the general formula for the homology of de Rham complexes we have an exact sequence

$$
0 \rightarrow \mathrm{SP}^{p^{n-1}} \rightarrow \mathrm{SP}^{p^{n}} \rightarrow I \rightarrow 0
$$

where the first map is Frobenius. Then by 2.4 we get the desired formula.

Now the proof of 4.3 consists of inserting 4.8, 4.9, 4.10 into formula 4.7.

4.11. Remark. It is easy to see that formula 4.4 gives us the full calculation of spectral sequences related to the de Rham complex. If we denote by $R_{n}$ the de Rham complex of length $p^{n}$ then (in the notation of Section 3 ) we see that ${ }^{1} E_{r, s}\left(R_{n}\right)$ has all differentials zero while in ${ }^{2} E_{r, s}\left(R_{n}\right)$ all possibly nontrivial differentials are embeddings.

4.12. REMARK (on the case $p=2$ ). If $p=2$ the situation is simpler than the one described above and the answer is slightly different, as is known from the classical results on the Steenrod algebra. Using the exact sequence (see [FLS, Section 2])

$$
\begin{aligned}
0 \rightarrow \mathrm{SP}^{2^{n-1}} \rightarrow \mathrm{SP}^{2^{n}} \rightarrow \mathrm{SP}^{2^{n}-1} \otimes \mathrm{SP}^{1} \rightarrow \ldots \rightarrow \mathrm{SP}^{2^{n}-i} \otimes \mathrm{SP}^{i} \rightarrow & \\
\ldots & \rightarrow \mathrm{SP}^{2^{n}} \rightarrow 0
\end{aligned}
$$

with the first map being the Frobenius one gets the formula

$$
\operatorname{Tor}_{j}^{\Gamma}\left(t, \mathrm{SP}^{2^{n}}\right)=\operatorname{Tor}_{j-2^{n}+1}^{\Gamma}\left(t, \mathrm{SP}^{2^{n}}\right) \oplus \operatorname{Tor}_{j-2^{n}}^{\Gamma}\left(t, \mathrm{SP}^{2^{n-1}}\right)
$$

and the rest of the calculation is left to the reader.

4.13. Remark (on cohomology). One can do similar calculations for the stable cohomology of $K(\mathbb{Z} / p, n)$. In this case, according to [P, Propo-

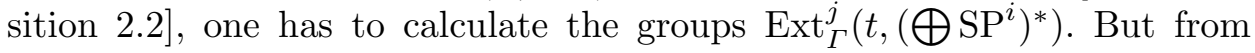
Koszul complexes one sees easily that for given $j$ only a finite number of $i$ 's have to be taken into account and hence, eventually, one has to compute 
the groups $\operatorname{Ext}_{\Gamma}^{j}\left(t,\left(\mathrm{SP}^{p^{i}}\right)^{*}\right)$. This can be done in precisely the same way as Tor calculations were obtained.

5. Remarks on the multiplicative structure. In [B2] Bousfield calculated the algebra structure on $L_{*}^{\text {st }}\left(\mathrm{SP}^{*}(\mathbb{Z} / p)\right)$ obtained from the composition multiplication of symmetric powers $\mathrm{SP}^{n} \circ \mathrm{SP}^{m} \rightarrow \mathrm{SP}^{n m}$. We want to understand how his algebra structure is related to the Steenrod algebra or its dual and this is the main subject of this section.

Let $T: \mathcal{V}_{p} \rightarrow \mathcal{V}_{p}$ be a covariant functor. Following [B2] we say that $T$ is a unital functor algebra if it comes equipped with two natural transformations $m: T \circ T \rightarrow T$ and $i: \mathrm{Id} \rightarrow T$ satisfying:

(i) (Associativity) The diagram

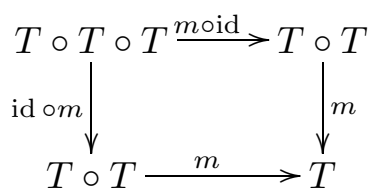

commutes.

(ii) (Unity) The compositions

$$
\text { Id } \circ T \stackrel{i \circ \text { id }}{\longrightarrow} T \circ T \stackrel{m}{\longrightarrow} T, \quad T \circ \mathrm{Id} \stackrel{\text { idoi }}{\longrightarrow} T \circ T \stackrel{m}{\longrightarrow} T
$$

are identities.

If $i$ does not exist then we talk about nonunital algebras. Similarly we define a functor coalgebra with $c: T \rightarrow T \circ T$ by reversing all the arrows in the definition above. Observe that if $T$ is a functor algebra then we have the following product structure (we will call it composition multiplication following Bousfield):

$$
\pi_{n}\left(T\left(\widetilde{\mathbb{Z}} / p\left[S^{m}\right]\right)\right) \times \pi_{m}\left(T\left(\widetilde{\mathbb{Z}} / p\left[S^{i}\right]\right)\right) \rightarrow \pi_{n}\left(T\left(\widetilde{\mathbb{Z}} / p\left[S^{i}\right]\right)\right) .
$$

The product takes $x \in \pi_{n}\left(T\left(\widetilde{R}\left[S^{m}\right]\right)\right)$ and $y \in \pi_{m}\left(T\left(\widetilde{R}\left[S^{i}\right]\right)\right)$ to $m_{*}(T(y) \circ x)$. Here we use the fact that if $A$ is any simplicial $\mathbb{Z} / p$-vector space then a pointed simplicial map $S^{n} \rightarrow A$ can be uniquely extended to a homomorphism $\widetilde{\mathbb{Z}} / p\left[S^{n}\right] \rightarrow A$.

If $T(0)=0$ then we can apply the stabilization procedure to get a product structure on the stable homotopy groups of $T$. Working with functor coalgebras we get a product structure on cohomotopy groups where by the $m$ th cohomotopy group of $T\left(\widetilde{\mathbb{Z}} / p\left[S^{n}\right]\right), \pi^{m}\left(T\left(\widetilde{\mathbb{Z}} / p\left[S^{n}\right]\right)\right)$, we mean the homotopy classes of homomorphisms $T\left(\widetilde{\mathbb{Z}} / p\left[S^{n}\right]\right) \rightarrow \widetilde{\mathbb{Z}} / p\left[S^{n}\right]$.

5.1. ExAmple. The functor $\mathbb{Z} / p[\cdot]$ has a coalgebra structure. The map $i: T \rightarrow$ Id is induced by the map $\mathbb{Z} / p[M] \rightarrow M$ which takes the formal sum 
to the sum in $M$. The map $c$ is induced by the extension of $r x \mapsto r(1 \cdot x)$. The same is true for the reduced functor.

5.2. Proposition. The composition multiplication obtained on the coalgebra $\widetilde{\mathbb{Z}} / p[\cdot]$ induces the standard multiplication of cohomology operations.

Proof. Let us write $K_{n}$ for the Eilenberg-MacLane space $K(\mathbb{Z} / p, n)$. We shall use $\widetilde{\mathbb{Z}} / p\left[S^{n}\right]$ as a model for $K_{n}$ and all (co)homology groups will have coefficients in $\mathbb{Z} / p$ if not specified otherwise. Every cohomology operation is determined by an element in $H^{*}\left(K_{n}\right)$ for some $n$ and hence by the homotopy class of maps $K_{n} \rightarrow K_{m}$. Multiplication of cohomology operations is equivalent to composition of maps. A map $f: K_{n} \rightarrow K_{m}$ induces a homomorphism $\mathbb{Z} / p(f): \widetilde{\mathbb{Z}} / p\left[K_{n}\right] \rightarrow K_{m}$ and the homotopy class of $f$ is uniquely determined by it. Let $f: K_{n} \rightarrow K_{m}$ and $g: K_{t} \rightarrow K_{n}$. We want to express $\mathbb{Z} / p(g \circ f)$ in terms of $\mathbb{Z} / p(f)$ and $\mathbb{Z} / p(g)$. It is easy to check working on generators that the homomorphism $\mathbb{Z} / p(g \circ f)$ is the same as $\mathbb{Z} / p(f) \circ \mathbb{Z} / p[\mathbb{Z} / p(g)] \circ c$ (the composition product of $[f]$ and $[g]$ coming from the coalgebra functor $\widetilde{\mathbb{Z}} / p[\cdot])$.

Observe that the functor $\mathbb{Z} / p[\cdot]$ has a nonunital algebra structure. It is given essentially by the augmentation map $\mathbb{Z} / p[A] \rightarrow A$ to which we apply $\mathbb{Z} / p[\cdot]$ for any $A \in \mathcal{A}_{R}$. We show:

5.3. Proposition. There are two algebra structures on both graded groups $H_{*}^{\text {st }}(K(\mathbb{Z}), \mathbb{Z} / p)$ and $H_{*}^{\text {st }}(K(\mathbb{Z} / p), \mathbb{Z} / p)$. One comes from the algebra stucture of $\mathbb{Z} / p[\cdot]$ and the other from composition of symmetric (reduced) powers and 1.1. These two structures are equivalent.

The main ingredient of the proof is Lemma 5.4 below. But before we formulate it we want to recall certain observations which go back to [S] and [DT]. Let $X$ be a simplicial set. Then $\mathrm{SP}^{\infty} X$ is a free simplicial abelian semigroup generated by $X$. The free simplicial abelian group $\mathbb{Z}[X]$ associated with $X$ can be treated as a group coming from the previous semigroup. The natural map $q: \operatorname{SP}^{\infty}(X) \rightarrow \widetilde{\mathbb{Z}}[X]$ is a homotopy equivalence.

5.4. Lemma. For any simplicial set $X$ the following diagram is homotopy commutative:

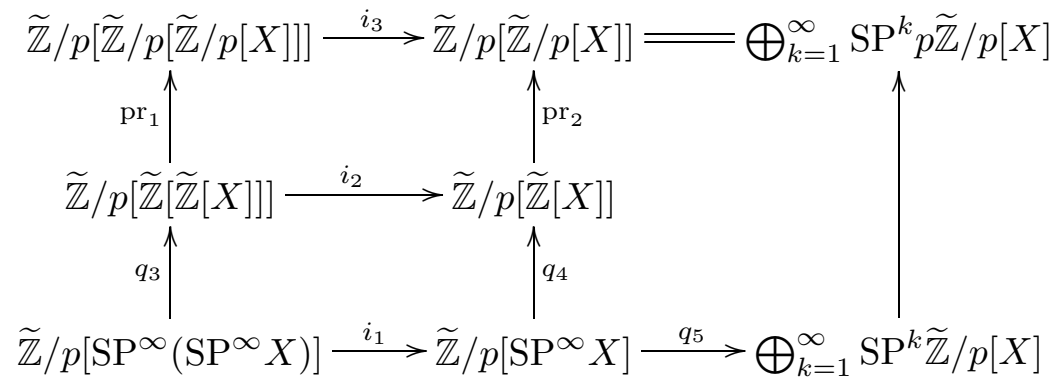


where $q_{i}$ is a general notation for the homotopy equivalence coming from 1.2 or $q: \mathrm{SP}^{\infty}(X) \rightarrow \mathbb{Z}[X], \mathrm{pr}_{j}$ denotes the maps coming from the natural projection $\mathbb{Z} \rightarrow \mathbb{Z} / p$, and the horizontal maps $i_{j}$ are essentially given by some version of the augmentation (the same which gives the algebra structure on $\mathbb{Z} / p[\cdot])$.

The proof of 5.4 is obvious from the definitions. We also have maps

$$
q_{1}: \bigoplus_{i=1}^{\infty} \mathrm{SP}^{i}\left(\bigoplus_{j=1}^{\infty} \mathrm{SP}^{j} \widetilde{\mathbb{Z}} / p[X]\right) \rightarrow \bigoplus_{i=1}^{\infty} \mathrm{SP}^{i}\left(\widetilde{\mathbb{Z}} / p\left[\operatorname{SP}^{\infty}(X)\right]\right)
$$

and

$$
g_{2}: \bigoplus_{i=1}^{\infty} \operatorname{SP}^{i}\left(\widetilde{\mathbb{Z}} / p\left[\operatorname{SP}^{\infty}(X)\right]\right) \rightarrow \widetilde{\mathbb{Z}} / p\left[\operatorname{SP}^{\infty}\left(\operatorname{SP}^{\infty} X\right)\right]
$$

which are defined the same way as the $q$-maps were defined before. Let now $X$ be a sphere of arbitrary dimension. Observe that $q_{5} \circ i_{1} \circ q_{2} \circ q_{1}$ is a map which gives us Bousfield's composition multiplication for $\sum_{i=1}^{\infty} \mathrm{SP}^{i}$. The map $i_{3}$ induces the algebra structure on $\mathbb{Z} / p[\cdot]$ and hence the algebra structure on $H_{*}^{\text {st }}(K(\mathbb{Z} / p), \mathbb{Z} / p)$. The map $\mathrm{pr}_{2}$ considered on stable homotopy gives us the embedding $H_{*}^{\text {st }}(K(\mathbb{Z}), \mathbb{Z} / p) \rightarrow H_{*}^{\text {st }}(K(\mathbb{Z} / p), \mathbb{Z} / p)$ from 1.3. The maps induced by pr on unstable and stable homotopy give us the desired comparison of the two algebra structures. A similar argument works for reduced symmetric powers.

As Bousfield calculated, the algebra structure from 5.3 is not equivalent to Milnor's dual to the Steenrod algebra. We are not going to do his calculations in our stable setting because we want to do them in the cohomological framework in another paper. Let us only mention some result on generators of the algebra structure.

5.5. Lemma. For any $i, \mathrm{SP}^{p^{i}}$ is a direct summand in $\mathrm{SP}^{p} \circ \mathrm{SP}^{p^{i-1}}$. The same is true for $\mathrm{SP}_{p}^{p^{i}}$.

Proof. There is a unique map $f: \mathrm{SP}^{r m} \rightarrow \mathrm{SP}^{r} \circ \mathrm{SP}^{m}$ which makes the following diagram commute:

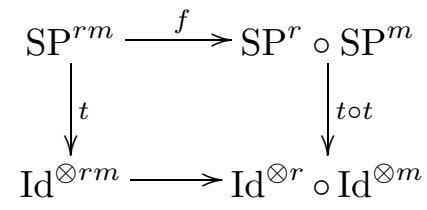

where $t: \mathrm{SP}^{i} \rightarrow \mathrm{Id}^{\otimes i}$ is the standard map

$$
t\left(x_{1} \cdot \ldots \cdot x_{i}\right)=\sum_{\tau \in \Sigma_{i}} x_{\tau(1)} \otimes \ldots \otimes x_{\tau(i)}
$$


and the lower horizontal map is given by inserting parentheses. The map $f$ is essentially given by sending a monomial of degree $\mathrm{rm}$ to the sum of monomials of degree $r$ in the variables given by all possible divisions of the set $\{i, \ldots, r m\}$ into sets of cardinality $m$ each. Now let $r=p$ and $m=p^{i-1}$. Then $f$ composed with the multiplication map $\mathrm{SP}^{p} \circ \mathrm{SP}^{p^{i-1}} \rightarrow \mathrm{SP}^{p^{i}}$ is an isomorphism. This is true because the number of monomials in the image of a monomial under $f$ is prime to $p$.

Lemma 5.5 is essentially contained in [B2, Section 8] though it is not stated separately. As an immediate corollary of 5.5 we obtain:

5.6. Corollary. The algebra $L_{*}^{\text {st }} \mathrm{SP}^{*}(\mathbb{Z} / p)$ with composition multiplication is generated by $L_{*}^{\mathrm{st}} \mathrm{SP}^{p}(\mathbb{Z} / p)$. Similarly, $L_{*}^{\mathrm{st}} \mathrm{SP}_{p}^{*}(\mathbb{Z} / p)$ is generated by $L_{*}^{\mathrm{st}} \mathrm{SP}_{p}^{p}(\mathbb{Z} / p)$.

5.7. Final Remark. Let $D^{i}$ denote the $i$ th divided power algebra functor. We know that $D^{i}(V)=\left(\operatorname{SP}^{i}\left(V^{*}\right)\right)^{*}$ for a finite-dimensional vector space $V$ and hence $\left(\mathrm{SP}^{i} \circ t^{*}\right)^{*}=D^{i} \circ t$ as functors of finite sets. It is easy to see that when we dualize $c$ from 5.2 we get a map of contravariant functors $c^{*}:\left(t^{*} \circ t^{*}\right)^{*} \rightarrow t$ which after applying both sides to the space $K(\mathbb{Z}, n)$ is equivalent to the map obtained from composition multiplication of divided power functors composed with $t$. So we can express multiplication of Steenrod operations in terms of the composition multiplication of divided powers. But of course it remains to get Adem relations in our stable framework. This problem will be addressed in a forthcoming paper.

\section{References}

[BS] S. Betley and J. Słomińska, New approach to the groups $H_{*}\left(\Sigma_{n}\right.$, Lie $\left._{n}\right)$ by the homology theory of the category of functors, J. Pure Appl. Algebra, to appear.

[B1] A. K. Bousfield, Homogeneous functors and their derived functors, mimeographed notes, 1967.

[B2] - Operations on derived functors of non-additive functors, preprint, 1967.

[DP] A. Dold und D. Puppe, Homologie nicht-additiver Funktoren. Anwendungen, Ann. Inst. Fourier (Grenoble) 11 (1961), 201-312.

[DT] A. Dold und R. Thom, Quasifaserungen und unendliche symmetrische Produkte, Ann. of Math. 67 (1958), 239-281.

[FLS] V. Franjou, J. Lannes et L. Schwartz, Autour de la cohomologie de MacLane des corps finis, Invent. Math. 115 (1994), 513-538.

[M] J. Milnor, The Steenrod algebra and its dual, Ann. of Math. 67 (1958), 150-171.

[P] T. Pirashvili, Hodge decomposition for higher order Hochschild homology, Ann. Sci. Ecole Norm. Sup. 33 (2000), 151-179. 
[S] E. Spanier, Infinite symmetric products, function spaces, and duality, Ann. of Math. 69 (1959), 142-198.

Institute of Mathematics

University of Warsaw

Banacha 2

02-097 Warszawa, Poland

E-mail: betley@mimuw.edu.pl

Received 14 September 2000;

in revised form 20 December 2000 